\title{
Modifying self-assembly and species separation in three-dimensional systems of shape-anisotropic particles
}

\author{
C. R. K. Windows-Yule,,${ }^{1,2, *}$ B. J. Scheper, ${ }^{1}$ W. K. den Otter, ${ }^{1,3}$ D. J. Parker ${ }^{2}$ and A. R. Thornton ${ }^{1}$ \\ ${ }^{1}$ Multiscale Mechanics (MSM), CTW and MESA+, University of Twente, P.O. Box 217, 7500 AE Enschede, The Netherlands \\ ${ }^{2}$ School of Physics and Astronomy, University of Birmingham, Edgbaston, Birmingham B15 2TT, United Kingdom \\ ${ }^{3}$ Computational Biophysics, TNW and MESA+, University of Twente, P.O. Box 217, 7500 AE Enschede, The Netherlands
}

(Received 29 May 2015; revised manuscript received 2 September 2015; published 4 February 2016)

\begin{abstract}
The behaviors of large, dynamic assemblies of macroscopic particles are of direct relevance to geophysical and industrial processes and may also be used as easily studied analogs to micro- or nano-scale systems, or model systems for microbiological, zoological, and even anthropological phenomena. We study vibrated mixtures of elongated particles, demonstrating that the inclusion of differing particle "species" may profoundly alter a system's dynamics and physical structure in various diverse manners. The phase behavior observed suggests that our system, despite its athermal nature, obeys a minimum free energy principle analogous to that observed for thermodynamic systems. We demonstrate that systems of exclusively spherical objects, which form the basis of numerous theoretical frameworks in many scientific disciplines, represent only a narrow region of a wide, multidimensional phase space. Thus, our results raise significant questions as to whether such models can accurately describe the behaviors of systems outside this highly specialized case.
\end{abstract}

DOI: 10.1103/PhysRevE.93.020901

Introduction. Arrays of athermal objects which interact solely through repulsive collisions can provide useful models for industrial processes [1], assemblies of microscopic particles [2], and even biological systems [3-8], reproducing complex natural phenomena, such as autonomous organization [9] or the potentially dangerous crowd-swarming of humans or animals [8]. We study beds of spherical and elongated particles rendered mobile through interactions with a vibrating surface. Where previous studies focus largely on shallow or quasitwo-dimensional systems and identical particles [2,4,5,10-13], we extend our investigation to fully three-dimensional binary mixtures, showing novel manners in which local and global ordering may be altered through variations in particle geometry. Using positron emission particle tracking (PEPT) [14], we are able to observe particle behaviors even deep within the large, opaque, and densely packed systems investigated $[15,16]$. Insights gained from the systems explored carry implications for various fields [1-9,17-20] and, with further study, may lead to a better ability to understand, and even control, numerous industrial and natural processes.

Experimental details. We study 11 distinct particle "species" with lengths $3.0 \leqslant l \leqslant 23.8 \mathrm{~mm}$, diameters $2.0 \leqslant$ $d \leqslant 5.0 \mathrm{~mm}$, and aspect ratios $p=\frac{l}{d} \in[1.0,11.9]$. The particles are carefully chosen to resemble one another as closely as possible: all are formed of chrome steel, ensuring equal density and surface properties, and a similar degree of triboelectric charging for all species; a spherocylindrical shape is chosen for elongated particles because spheres can be considered a special case of this geometry; finally, the chosen geometries also ensure consistent convexity and angularity, properties known to affect system dynamics [21,22]. The large particle mass negates air effects [23]. Particles are housed in a $120 \mathrm{~mm}$ square-based cuboidal container of height $200 \mathrm{~mm}$. Unless stated otherwise, all data presented pertain to this system geometry. The system is vibrated vertically, providing

*Corresponding author: windowsyule@gmail.com energy to the particles. Various combinations of frequency, $f \in$ $[11.4,216.0] \mathrm{Hz}$, and amplitude, $A \in[0.05,0.96] \mathrm{mm}$, are used to provide a range of peak driving velocities, $v=2 \pi f A \in$ $[0.02,0.42] \mathrm{ms}^{-1}$. Although the dimensionless acceleration, $\Gamma=\frac{4 \pi^{2} f^{2} A}{g}$, determines the onset of surface motion in a vibrated system [24-26], the onset of full fluidization is characterized by $v$ [27-29], which we take as our key control parameter. Preliminary experiments show the system behavior to be independent of the specific combinations of $f$ and $A$ used to produce a given $v$. Our systems are investigated using PEPT, a noninvasive technique capable of analyzing, with submillimeter accuracy and millisecond-scale temporal resolution [14], the dynamics of particulate systems in all spatial dimensions. A single particle, identical to all others of its species, is radioactively labeled with ${ }^{55} \mathrm{Co}$, producing back-to-back $\gamma$ rays which allow its motion to be tracked [14,30]. Through appropriate time averaging [15], the tracer's motion may be used to extract information pertaining to the system as a whole, including residence profiles [15] and mean-squared displacements [31], two quantities specifically explored here. Although not necessary to the understanding of this Rapid Communication, the interested reader may find information regarding the PEPT technique and the manners in which it may be applied to produce whole-field data in mono-, bi- or polydisperse systems in Refs. [14], [15,31], and $[16,32,33]$, respectively. Residence profiles are produced from PEPT data by recording the system's steady state for a period $\geqslant 10800 \mathrm{~s}$ at constant $v$ and plotting time-averaged results corresponding to the system's spatial distribution of particles for pairs of repeated experiments. While there exists a constant flux of particles into and out of any given region of the system, the layers formed remain constant, allowing accurate profiles to be produced.

Results. Figure 1 shows examples of the diverse states produced by differing particle combinations. The states of our systems are found to be dependent on both the relative particle length $\frac{l_{1}}{l_{2}}$ and diameter $\frac{d_{1}}{d_{2}}$, as well as on individual species' 


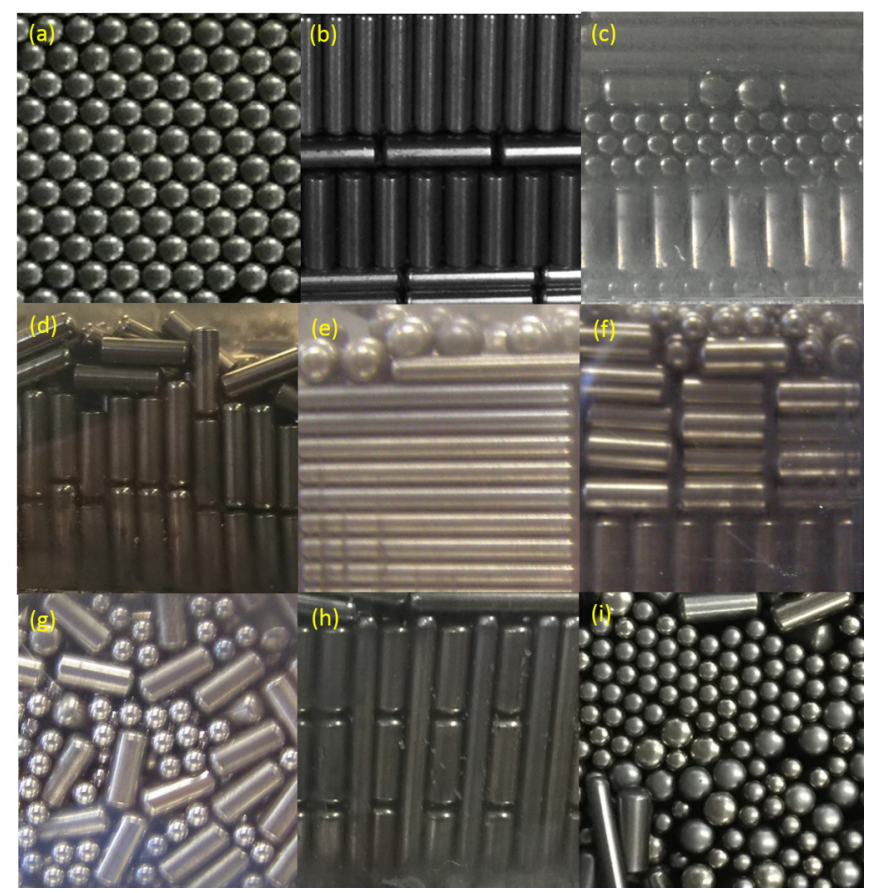

FIG. 1. Typical particle ordering in side views (b)-(h) or top views (a) and (i) for (a) any single-species region of vertically aligned cylinders; binary systems with $d_{1} \neq d_{2}$ for particles with (b) similar $p$ and (c) strongly differing $p$; (d) binary systems with $d_{1}=d_{2}$; sphere-rod mixtures with (e) $d_{\text {rod }}<d_{\text {sphere }}$ and (f) $d_{\text {rod }}>d_{\text {sphere }}$; (g) system in (f) with a lower concentration of cylinders $\left[\phi_{c}=0.5\right.$ as opposed to $\phi_{c}=0.9$ for (e) and (f)]; (h) and (i) same system as (c) in a cylindrical container. All rod-rod systems shown comprise two vertical HCP layers of particles.

aspect ratios $p_{1}$ and $p_{2}$. The multidimensional parameter space to which our system is subject causes an overlap of distinct states for any two-dimensional (2D) representations. As such, in Fig. 2, we show two individual 2D projections on the $\frac{l_{1}}{l_{2}}-\frac{d_{1}}{d_{2}}$ and $p_{1}-p_{2}$ planes such that the influence of all parameters can be clearly observed. All data in this figure were acquired using a number of particles equivalent to two hexagonal close-packed (HCP) layers of vertically aligned particles for each species present. Since the total number $N$ and mass of particles will vary for different binary mixtures, a fixed driving strength $v$ will produce differing degrees of excitation for the various systems; thus, for consistency, each system was driven using the minimal $v$ for which full fluidization was observed.

(i) Description of the states. The configurations formed by our system can be categorized into six main states. State A corresponds to a purely spherical (PS) system exhibiting the well-known size segregation [34-37] whereby large (small) particles of equal density migrate to higher (lower) regions, with no local ordering. In state $\mathrm{B}$, we observe microphase separation: particles segregate vertically, forming distinct bands, each comprising only a single species, displaying strong alignment [Fig. 1(b)]. We denote $\mathrm{C}$ as a subset of state $\mathrm{B}$ in which species with large aspect ratios demonstrate exclusively horizontal alignment [Fig. 1(c)]. In state D, particles align vertically with no clear banding [Fig. 1(d)]. While states A-D are seemingly insensitive to the relative volume
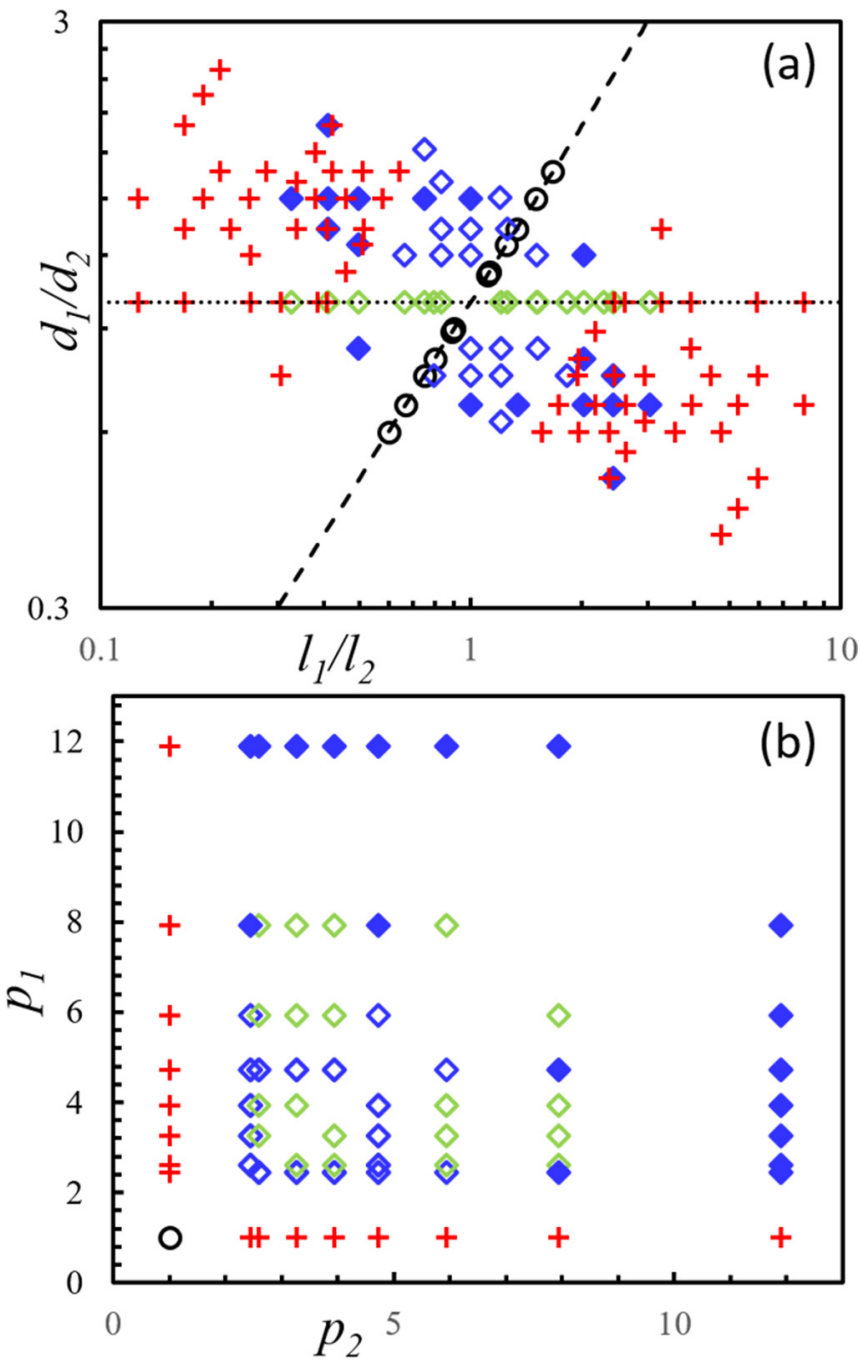

FIG. 2. Two-dimensional projections of the phase diagram showing for a range of binary mixtures of spheres and/or spherocylinders, the ordered states produced for each combination of (a) relative particle diameter $\left(\frac{d_{1}}{d_{2}}\right)$ and length $\left(\frac{l_{1}}{l_{2}}\right)$ and (b) the aspect ratios $p_{1,2}$ of the differing constituents. State A: open circles; state B: open blue diamonds; state $\mathrm{C}$ : solid blue diamonds; state D: green diamonds; state E: crosses. The dashed and dotted lines in (a) show, respectively, the regions of phase space for which $d_{1}=d_{2}$ and $\frac{d_{1}}{d_{2}}=\frac{l_{1}}{l_{2}}$. All states are defined in the main text.

fraction, $\phi_{i}=\frac{N_{i} V_{i}}{\sum_{j} N_{j} V_{j}}$, of a given species $i$ (with similar configurations observed for $0.1 \leqslant \phi_{i} \leqslant 0.9$ ), for the special case of rod-sphere mixtures $\left(p_{1 \mid 2}=1\right)$, we see an additional $\phi_{i}$ dependence. For high cylinder volume fractions $\phi_{c}$, we observe state E, comprising an ordered lower region of rods showing horizontal and/or vertical alignment, and a disordered upper region comprising all spheres and any cylindrical particles excluded from the lower bed [Figs. 1(e) and 1(f)]. As $\phi_{c}$ is decreased, we see a transition to a disordered, mixed state $\mathrm{F}$ [Fig. 1(g)]. The value of $\phi_{c}$ at which this transition occurs is dependent on the strength $v$ with which the system is driven [see Fig. 3(a)]. The addition of spherical particles is also found to alter the system dynamics, producing a transition, under otherwise identical conditions, from the slow, glassy 

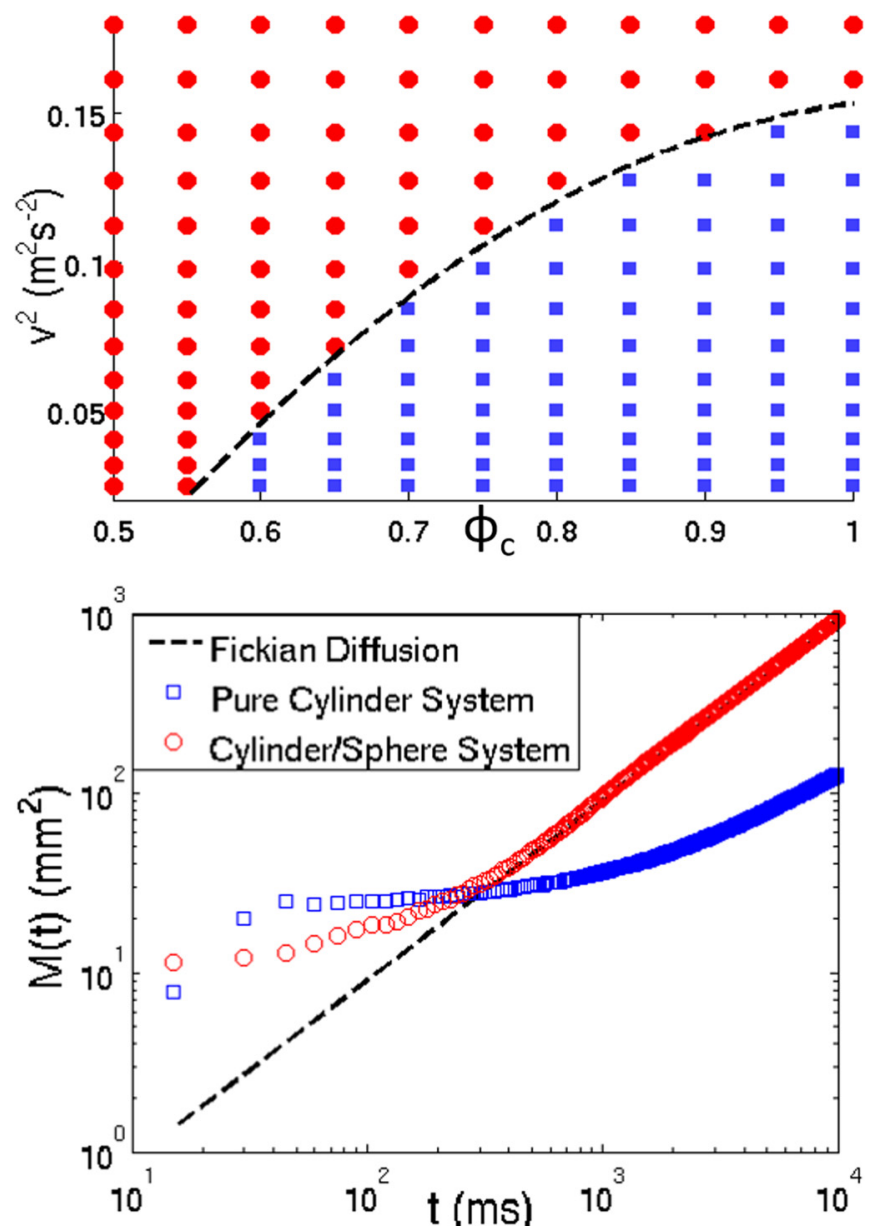

FIG. 3. Above: Phase diagram showing the regions of $v^{2}-\phi_{c}$ space for which systems of $4 \mathrm{~mm}$ spheres and $9.8 \times 3 \mathrm{~mm}$ cylinders form the ordered, segregated state $\mathrm{E}$ (blue squares) and disordered, mixed state F (red circles). The dotted line approximates the system's phase boundary. For $v^{2}<0.02 \mathrm{~m}^{2} \mathrm{~s}^{-2}$, particle rearrangement is suppressed because the system is no longer fluidized. Below: Mean-squared displacement $M(t)$ for the cylinders in a monodisperse system $\left(\phi_{c}=1, v=0.27\right.$, state E) and an equivalent cylinder and sphere system $\left(\phi_{c}=0.75, v=0.27\right.$, state $\left.F\right)$; the system "doped" with spheres approximates Fickian diffusion, while the "pure" system demonstrates subdiffusive behavior, indicating glassy dynamics [47].

dynamics associated with jamming [38-40] to faster, fluid-like dynamics. This is demonstrated by the differing slope $[39,41]$ of the mean-squared displacement for the pure-cylinder and cylinder-sphere cases, as shown in Fig. 3(b).

(ii) Limitations of spherical models. The majority of existing studies and theoretical frameworks concerning particulate media focus on assemblies of exclusively spherical particles $[42,43]$, as do numerous simulational and theoretical models in other disciplines, e.g., colloidal physics [44], astrophysics [45], and polymer science [46]. However, Fig. 2 shows that such systems correspond to only a single line in an expansive parameter space, highlighting the strong influence of geometric effects on the behaviors of discrete systems. As we deviate even slightly from this well-explored line, we observe various states which are unobserved in spherical assemblies, and behaviors which directly contradict our expectations based
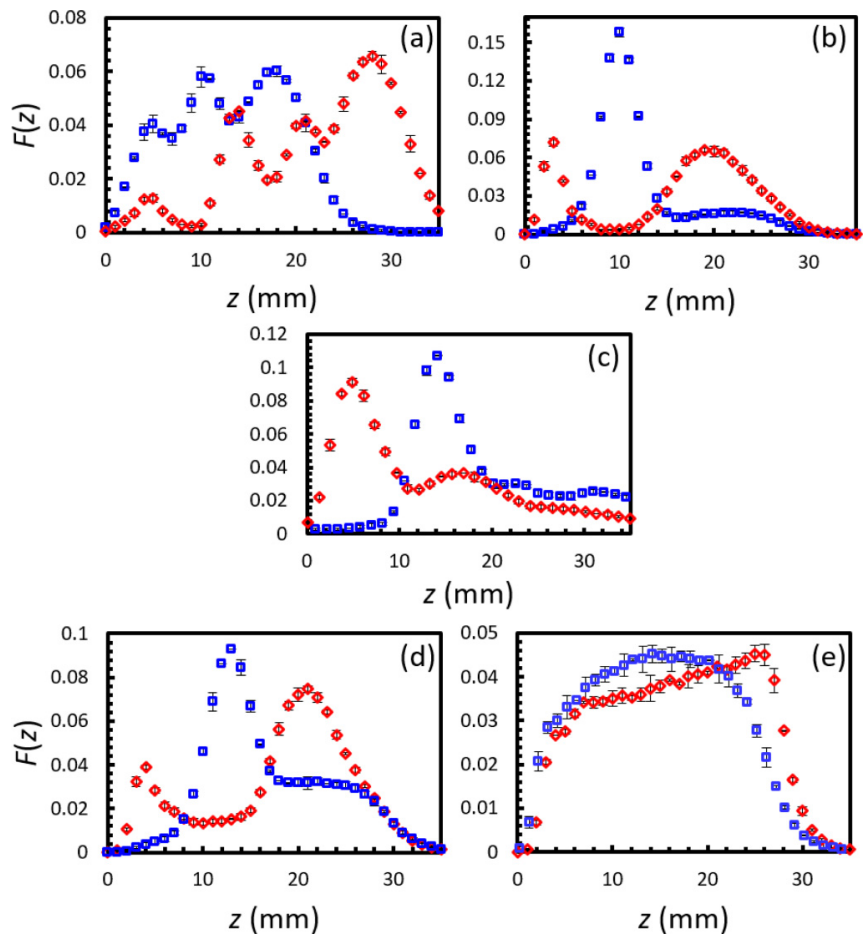

FIG. 4. One-dimensional residence profiles for various binary systems. Each residence profile $F(z)$ can be considered as a histogram of the height of particles for a given species [15]. Data are shown for binary combinations of (a) $9.8 \times 3$ and $7.8 \times 3 \mathrm{~mm}$ rods, (b) $23.8 \times 2$ and $9.8 \times 3 \mathrm{~mm}$ rods, (c) $9.8 \times 4 \mathrm{~mm}$ rods and $3 \mathrm{~mm}$ spheres $\left(\phi_{c}=0.6\right)$, (d) $23.8 \times 2$ and $7.8 \times 3 \mathrm{~mm}$ rods, and (e) 4 and $4.5 \mathrm{~mm}$ spheres. In all cases, longer spherocylinders (larger spheres) are represented by diamonds and shorter (smaller) species by squares. For all pure-sphere and pure-cylinder systems, equal volumes of each species are used $\left(\phi_{i}=0.5\right)$.

on PS systems in terms of both the strength and direction of segregation. For instance, Fig. 4 shows that for nonspherical particles, conventional size segregation will not consistently occur based on any measure of size, including particle radius, length, or volume $V$. The most remarkable example is perhaps Fig. 4(c), where particles which are objectively larger in all aspects $(l, d$, and $V)$ segregate downward, in direct contradiction of our expectations based on systems of spheres. Figures 4(d) and 4(e) show a marked increase in segregation for the cylindrical system despite the smaller volume ratio $\frac{V_{1}}{V_{2}}$ between its components as compared to the PS system.

Discussion and analysis. The states and dynamics of athermal particulate systems such as those studied here are notoriously poorly understood $[48,49]$ with, at present, no accepted theory to explain or predict their behaviors. In thermodynamic systems such as colloidal suspensions, phase behavior is determined by the minimization of free energy [50-52], $F=U-T S$, with $T$ the system temperature, $S$ its entropy, and $U$ internal energy. We propose that the major findings of this Rapid Communication can be explained through an analogy to the principle of minimum free energy, with the peak vibrational velocity acting as an effective temperature. (i.e., $T \propto v^{2}$ ) [26,53]. 
(i) Ordered states. In rod-rod systems, an ordered configuration is favorable for two reasons: first, the higher packing density $\eta$ leads to a lower mass center and hence lower gravitational potential energy, thus minimizing $U$, a mechanism already proposed to influence segregative processes in nonspherical materials [54]. Second, for relatively dense systems, particle alignment also acts to increase entropy [55] by allowing the system more translational freedom relative to an equally dense disordered system. The specific arrangement achieved seemingly depends on the degree to which a given configuration can minimize $U$; i.e., maximize $\eta$. For instance, since rods with differing diameters cannot pack as efficiently as rods with equal $d$ [56], a binary system with $d_{1} \neq d_{2}$ will form distinct layers of $d_{1}$ and $d_{2}$ cylinders (state B) in order to maximize $\eta$. A binary system with $d_{1}=d_{2}$ but $l_{1} \neq l_{2}$, meanwhile, can maximize $\eta$ by forming a columnar structure with no distinct layers (state D). Perhaps the clearest indication of our systems' tendencies to maximize $\eta$ is provided by studying a system comprising long $23 \times 2 \mathrm{~mm}$ and short $9.8 \times 3 \mathrm{~mm}$ rods, with a number of long rods adequate to produce multiple horizontal layers, but too small to produce a full vertically aligned layer. Clearly, the optimal arrangement in our cuboidal container is for the system to form a layered arrangement of short particles and horizontally aligned long particles [state C, Fig. 1(d) [57]]. If, however, we house a similar particle assembly in a cylindrical container, the inability to form a defect-free horizontal packing means that the formation of mixed, solely vertically aligned layers now becomes energetically favorable over state $\mathrm{C}$. The result is an ordered arrangement such as that shown in Fig. 1(h), where longer vertically aligned particles coexist with stacks of shorter particles - an elegant example of a "mindless" system solving a nontrivial energy minimization problem. The preference of purely vertical (as opposed to horizontal) alignment for elongated particles housed in cylindrical systems has been previously observed in both experiments [10] and simulations [58] of monodisperse systems, again in agreement with our expectations based on energy minimization. While systems of rods can achieve near-ideal packing [11,13], PS systems under simple vibration cannot achieve the energetically optimal [59] face-centred cubic arrangement [60-62]. Thus, free energy in a PS system is minimized by increasing $S$ as opposed to decreasing $U$. For binary systems of spheres, the free volume available to particles is higher in segregated, as opposed to mixed, states [63] making such a state (i.e., state A) entropically favorable [64]. The specific arrangement (large particles above small) can be explained by the presence of gravity [65]; in systems where $g$ is not important, the direction of segregation is arbitrary, as expected from thermodynamics [66].

(ii) Phase transitions in sphere-cylinder systems. The principle of free-energy minimization can also explain the more complex state $\mathrm{E}$, and its transition from local order and global segregation to the isotropic mixed state $\mathrm{F}$ with increasing $v$ and/or decreasing $\phi_{c}$. Consider first the limiting case $\phi_{c} \rightarrow 1$. Here, as we have seen, an ordered state is energetically and entropically favorable. However, for an adequately large number of spheres, a disordered mixed state will give a net increase in $S$ compared to an equivalent ordered state, as spheres will be free to explore the entire system, as opposed to being constrained to a limited region [cf. Figs. 1(f) and $1(\mathrm{~g})]$ and rotational freedom becomes entropically important for cylinders $[55,67]$. This alone, however, is not enough to guarantee a phase transition, as the disordered mixed state will also decrease $\eta$ and hence increase $U$. If our system is indeed governed by free energy minimization, the order-to-disorder transition will also require an adequately high $T$ (i.e., large $v$ ) such that $(U-T S)_{\text {disordered }}<(U-T S)_{\text {ordered }}$. In short, based on free energy minimization arguments, we would expect the following to hold: firstly for fixed $v$ (i.e., fixed $T$ ), an increase in the volume fraction $\phi_{c}$ of cylinders should lead to a transition from disorder to order, as is indeed observed in colloidal systems [68]. Second, an increase in $v$ for fixed $\phi_{c}$ should also result in a transition from order to disorder. Finally, there should exist a value of $v$ above which our system is mixed and disordered for all $\phi_{c}$, an analog to the upper critical solution temperature in thermodynamic systems. Figure 3(a) agrees well with these expectations, sharing key features with the temperature-composition phase diagrams of various colloidal, polymeric, and liquid crystal systems $[69,70]$.

Order-disorder transitions may occur even for $\phi_{c}=1$; for large $v$, the bed will expand significantly, lowering $\eta$ and allowing rods to rotate more freely while also eliminating the potential-energy-reducing effects of alignment, thus making disorder favorable. This again provides a parallel with thermodynamic expectations, where the Onsager theory [67] dictates that a system's state is a function both of $p$ and $\eta$. With this in mind, it is interesting to consider the limit $p \rightarrow 1$, i.e., the transition from spherocylinders to spheres. In agreement with previous studies [11] we observe that the order-disorder transition shifts to lower $v$ with decreasing $p$. For spheres, the transition shifts below the minimum $v$ required for fluidization, i.e., ordered packing is never favorable for spheres.

Our results suggest that the system configurations observed correspond to global, as opposed to local, minima in state F, since repeated experiments starting from randomized initial conditions invariably provide the same final configuration (or, more precisely, an equivalent degenerate microstate corresponding to the same macrostate).

The apparent applicability of thermodynamic concepts to macroscopic systems is perhaps surprising, yet not unfeasible. Prior works also suggest such a possibility for systems such as ours which, though in nonequilibrium, exist in a steady state and are subject to an analog to temperature [71]. These studies, however, are typically restricted to constrained, quasi-two-dimensional systems and/or assemblies of spheres [72-79], the limitations of which we have already discussed. The systems studied here are closer in size, dimensionality, and complexity to real-world systems, providing an important indicator that such a theoretical framework is also likely to be applicable to many large-scale industrial and natural processes which, at present, remain poorly understood [48]. Conversely, the existence of macroscopic systems which obey thermodynamic-like laws may also prove valuable as analogs for microscopic systems, whose direct study may prove challenging. For instance, the ability to produce specific structures simply by manipulating energy and entropy via particle shape, i.e., "building order from disorder" [80], shows strong parallels with work performed in colloidal 
systems [52]. Our systems may even serve as useful, easily studied models for biological systems [4,5]. For example, our findings regarding the suppression of self-organization and the dispersion of localized conglomerations of a given species may have relevance [7] in preventing the swarming of bacteria, a behavior shown to increase adaptive antibiotic resistance [81] — one of the great problems facing society today.

Conclusions. In this Rapid Communication, we have provided insight into a number of largely unexplored states accessible to three-dimensional binary assemblies of macroscopic spheres and spherocylinders, demonstrating the limitations of our current understanding of particulate systems based predominantly on studies of exclusively spherical particles. We have shown that minor alterations to a system's composition can invoke dramatic alterations in its state and dynamics, including the transition from phase separation to mixing, and the frustration of "jamming" [17] effects, two observations of potential relevance to a broad range of industrial processes [82-85]. All of our findings have been explained in the context of free energy minimization, suggesting that an analog to classical thermodynamics may be applicable even to large three-dimensional systems of dynamic athermal and aspherical particles.

Acknowledgments. The authors would like to express their gratitude to the Hawkesworth Family Trust whose support through a Hawkesworth Scholarship has made this work possible. This study was supported by the Nederlandse Organisatie voor Wetenschappelijk Onderzoek (NWO), through the Dutch Technology Foundation, Stichting voor de Technische Wetenschappen (STW) via Project 13472.
[1] J. Duran, Sands, Powders, and Grains (Springer, New York, 2000).

[2] J. Galanis, D. Harries, D. L. Sackett, W. Losert, and R. Nossal, Phys. Rev. Lett. 96, 028002 (2006).

[3] D. Helbing, L. Buzna, A. Johansson, and T. Werner, Transport. Sci. 39, 1 (2005).

[4] S. Mishra and S. Ramaswamy, Phys. Rev. Lett. 97, 090602 (2006).

[5] V. Narayan, S. Ramaswamy, and N. Menon, Science 317, 105 (2007).

[6] M. Moussaï, D. Helbing, and G. Theraulaz, Proc. Nat. Acad. Sci. U.S.A. 108, 6884 (2011).

[7] S. R. McCandlish, A. Baskaran, and M. F. Hagan, Soft Matter 8, 2527 (2012).

[8] I. Zuriguel, D. R. Parisi, R. C. Hidalgo, C. Lozano, A. Janda, P. A. Gago, J. P. Peralta, L. M. Ferrer, L. A. Pugnaloni, E. Clément et al., Sci. Rep. 4, 7324 (2014).

[9] G. M. Whitesides and B. Grzybowski, Science 295, 2418 (2002).

[10] F. X. Villarruel, B. E. Lauderdale, D. M. Mueth, and H. M. Jaeger, Phys. Rev. E 61, 6914 (2000).

[11] M. Ramaioli, L. Pournin, and T. M. Liebling, Phys. Rev. E 76, 021304 (2007).

[12] A. Kudrolli, G. Lumay, D. Volfson, and L. S. Tsimring, Phys. Rev. Lett. 100, 058001 (2008).

[13] V. Yadav, J.-Y. Chastaing, and A. Kudrolli, Phys. Rev. E 88, 052203 (2013).

[14] D. Parker, R. Forster, P. Fowles, and P. Takhar, Nucl. Instrum. Methods Phys. Res., Sect. A 477, 540 (2002).

[15] R. D. Wildman, J. Huntley, J. P. Hansen, D. J. Parker, and D. A. Allen, Phys. Rev. E 62, 3826 (2000).

[16] R. D. Wildman and D. J. Parker, Phys. Rev. Lett. 88, 064301 (2002).

[17] A. J. Liu and S. R. Nagel, Nature 396, 21 (1998).

[18] S. Luding, Nature 435, 159 (2005).

[19] T. Shinbrot and F. Muzzio, Encyclopedia of Pharmaceutical Technology (CRC Press, Boca Raton, 2002).

[20] K. R. LaMarche, M. J. Metzger, B. J. Glasser, and T. Shinbrot, Phys. Rev. E 81, 052301 (2010).

[21] E. Azéma, N. Estrada, and F. Radjai, Phys. Rev. E 86, 041301 (2012).
[22] N. Gravish, S. V. Franklin, D. L. Hu, and D. I. Goldman, Phys. Rev. Lett. 108, 208001 (2012).

[23] C. Zeilstra, M. A. van der Hoef, and J. A. M. Kuipers, Phys. Rev. E 77, 031309 (2008).

[24] P. Evesque and J. Rajchenbach, Phys. Rev. Lett. 62, 44 (1989).

[25] P. Philippe and D. Bideau, Phys. Rev. Lett. 91, 104302 (2003).

[26] J. A. Dijksman and M. van Hecke, Europhys. Lett. 88, 44001 (2009).

[27] E. Clement and J. Rajchenbach, Europhys. Lett. 16, 133 (1991).

[28] J. Gallas, H. Herrmann, and S. Sokołowski, Physica A (Amsterdam) 189, 437 (1992).

[29] A. Götzendorfer, C.-H. Tai, C. A. Kruelle, I. Rehberg, and S.-S. Hsiau, Phys. Rev. E 74, 011304 (2006).

[30] D. Parker, C. Broadbent, P. Fowles, M. Hawkesworth, and P. McNeil, Nucl. Instrum. Methods Phys. Res., Sect. A 326, 592 (1993).

[31] R. D. Wildman, J. M. Huntley, and D. J. Parker, Phys. Rev. E 63, 061311 (2001).

[32] C. R.K. Windows-Yule, T. Weinhart, D. J. Parker, and A. R. Thornton, Phys. Rev. Lett. 112, 098001 (2014).

[33] C. Windows-Yule, A. Rosato, N. Rivas, and D. Parker, New J. Phys. 16, 063016 (2014).

[34] A. Rosato, K. J. Strandburg, F. Prinz, and R. H. Swendsen, Phys. Rev. Lett. 58, 1038 (1987).

[35] J. B. Knight, H. M. Jaeger, and S. R. Nagel, Phys. Rev. Lett. 70, 3728 (1993).

[36] J. Duran, J. Rajchenbach, and E. Clément, Phys. Rev. Lett. 70, 2431 (1993).

[37] D. A. Huerta and J. C. Ruiz-Suárez, Phys. Rev. Lett. 92, 114301 (2004).

[38] L. E. Silbert, D. Ertaş, G. S. Grest, T. C. Halsey, and D. Levine, Phys. Rev. E 65, 051307 (2002).

[39] G. Marty and O. Dauchot, Phys. Rev. Lett. 94, 015701 (2005).

[40] A. R. Abate and D. J. Durian, Phys. Rev. E 74, 031308 (2006).

[41] B. Utter and R. P. Behringer, Phys. Rev. E 69, 031308 (2004).

[42] J. Ottino and D. Khakhar, Annu. Rev. Fluid Mech. 32, 55 (2000).

[43] R. Caulkin, X. Jia, M. Fairweather, and R. A. Williams, Phys. Rev. E 81, 051302 (2010).

[44] E. J. Meijer and D. Frenkel, Phys. Rev. Lett. 67, 1110 (1991). 
[45] A. Bodrova, J. Schmidt, F. Spahn, and N. Brilliantov, Icarus 218, 60 (2012).

[46] J. A. Torres, P. F. Nealey, and J. J. De Pablo, Phys. Rev. Lett. 85, 3221 (2000).

[47] A. S. Keys, A. R. Abate, S. C. Glotzer, and D. J. Durian, Nat. Phys. 3, 260 (2007).

[48] H. M. Jaeger, S. R. Nagel, and R. P. Behringer, Rev. Mod. Phys. 68, 1259 (1996).

[49] F. J. Muzzio, T. Shinbrot, and B. J. Glasser, Powder Technol. 124, 1 (2002).

[50] D. A. McQuarrie, Statistical Thermodynamics (Harper Collins, New York, 1973).

[51] D. J. Wales and T. V. Bogdan, J. Phys. Chem. B 110, 20765 (2006).

[52] M. Adams, Z. Dogic, S. L. Keller, and S. Fraden, Nature 393, 349 (1998).

[53] J.-C. Géminard and C. Laroche, Phys. Rev. E 68, 031305 (2003).

[54] C. R. Abreu, F. W. Tavares, and M. Castier, Powder Technol. 134, 167 (2003).

[55] P. J. Flory, in Proceedings of the Royal Society of London A: Mathematical, Physical and Engineering Sciences, Vol. 234 (The Royal Society, London, 1956), pp. 73-89.

[56] R. van Elst, Sphere packings of discs with two radii, http://alexandria.tue.nl/extra1/afstversl/wsk-i/elst2012.pdf.

[57] Note that for $p$ values which lie far from unity, state $\mathrm{C}$ remains favorable even if there exist enough longer particles to form a full vertical layer, because the energy [11] and geometrical rearrangements required to form such a layer of long particles render such a configuration unfavorable compared to the formation of multiple horizontal layers. For geometrical arrangements, see E. Caglioti, V. Loreto, H. J. Herrmann, and M. Nicodemi, Phys. Rev. Lett. 79, 1575 (1997).

[58] L. Pournin, M. Weber, M. Tsukahara, J.-A. Ferrez, M. Ramaioli, and T. M. Liebling, Granular Matter 7, 119 (2005).

[59] S.-C. Mau and D. A. Huse, Phys. Rev. E 59, 4396 (1999).

[60] J. B. Knight, C. G. Fandrich, C. N. Lau, H. M. Jaeger, and S. R. Nagel, Phys. Rev. E 51, 3957 (1995).

[61] M. Nicodemi, A. Coniglio, and H. J. Herrmann, Phys. Rev. E 55, 3962 (1997).

[62] Y. Nahmad-Molinari and J. C. Ruiz-Suarez, Phys. Rev. Lett. 89, 264302 (2002).
[63] A. Yu and N. Standish, Powder Technol. 55, 171 (1988).

[64] A. Kudrolli, Rep. Prog. Phys. 67, 209 (2004).

[65] S. Savage and C. Lun, J. Fluid Mech. 189, 311 (1988).

[66] P. M. Reis and T. Mullin, Phys. Rev. Lett. 89, 244301 (2002).

[67] L. Onsager, Ann. N.Y. Acad. Sci. 51, 627 (1949).

[68] G. A. Vliegenthart, A. van Blaaderen, and H. N. W. Lekkerkerker, Faraday Discuss. 112, 173 (1999).

[69] F. Benmouna, L. Bedjaoui, U. Maschke, X. Coqueret, and M. Benmouna, Macromol. Theory Simul. 7, 599 (1998).

[70] H. Frielinghaus, N. Hermsdorf, K. Almdal, K. Mortensen, L. Messé, L. Corvazier, J. Fairclough, A. Ryan, P. Olmsted, and I. Hamley, Europhys. Lett. 53, 680 (2001).

[71] S. Ogawa, in Proceedings of the U. S.-Japan Seminar on Continuum Mechanics and Statistical Approaches in the Mechanics of Granular Materials, edited by S. C. Cowin and M. Satake (Gakujutsu Bunken Fukyu-Kai, Tokyo, 1978), pp. 208-217.

[72] M. Nicodemi, Phys. Rev. Lett. 82, 3734 (1999).

[73] M. Argentina, M. G. Clerc, and R. Soto, Phys. Rev. Lett. 89, 044301 (2002).

[74] F. Cecconi, F. Diotallevi, U. M. B. Marconi, and A. Puglisi, J. Chem. Phys. 120, 35 (2004).

[75] Y. Martínez-Ratón, Phys. Rev. E 75, 051708 (2007).

[76] R. Liu, Y. Li, M. Hou, and B. Meerson, Phys. Rev. E 75, 061304 (2007).

[77] J. Galanis, R. Nossal, and D. Harries, Soft Matter 6, 1026 (2010).

[78] K. Roeller, J. P. D. Clewett, R. M. Bowley, S. Herminghaus, and M. R. Swift, Phys. Rev. Lett. 107, 048002 (2011).

[79] J. P. Clewett, J. Wade, R. Bowley, S. Herminghaus, M. R. Swift, and M. G. Mazza, arXiv:1503.00159.

[80] D. Frenkel, in Complex Fluids (Springer, New York, 1993), pp. 137-148.

[81] J. Overhage, M. Bains, M. D. Brazas, and R. E. Hancock, J. Bacteriol. 190, 2671 (2008).

[82] S. Satija and I. Zucker, Drying Technol. 4, 19 (1986).

[83] A. D. Rosato, D. L. Blackmore, N. Zhang, and Y. Lan, Chem. Eng. Sci. 57, 265 (2002).

[84] S.-S. Hsiau, C.-C. Liao, P.-Y. Sheng, and S.-C. Tai, Exp. Fluids 51, 795 (2011).

[85] A. J. Liu and S. R. Nagel, Soft Matter 6, 2869 (2010). 